EOMmUn: Communication et organisation

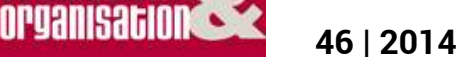

Design \& projet

\title{
Le dispositif Design-Projet : vers une temporalité par l'usage
}

Rihab Zaidi

\section{(2) OpenEdition}

1 Journals

Édition électronique

URL : http://journals.openedition.org/communicationorganisation/4726

DOI : 10.4000/communicationorganisation.4726

ISBN : 979-10-300-0155-6

ISSN : 1775-3546

Éditeur

Presses universitaires de Bordeaux

Édition imprimée

Date de publication : 1 décembre 2014

Pagination : 43-56

ISBN : 978-2-86781-905-6

ISSN : 1168-5549

\section{Référence électronique}

Rihab Zaidi, «Le dispositif Design-Projet : vers une temporalité par l'usage », Communication et

organisation [En ligne], 46 | 2014, mis en ligne le 01 décembre 2017, consulté le 26 avril 2019. URL :

http://journals.openedition.org/communicationorganisation/4726 ; DOI : 10.4000/

communicationorganisation. 4726 


\title{
Le dispositif Design-Projet : vers une temporalité par l'usage
}

\author{
Rihab Zaidi ${ }^{1}$
}

\section{Introduction}

Le design contemporain est de plus en plus appelé à repenser l'incertain et le variable pour redessiner un mode de vie approprié à un contexte social en perpétuelle mutation. Désormais, il est tenu d'apporter un regard critique sur un monde pensé en tant qu'objet et non pas contexte de vie. Il réfléchit sur un devenir, déplace les limites des usages et propose de nouvelles stratégies de résolution de problème de notre quotidien. Pour ce faire, il s'inscrit dans un projet.

Nous souhaitons quitter cette espèce de lieu commun où design et projet sont hantés par la vitesse et l'actualité pour essayer de redéfinir le rapport designprojet à partir de notions telles que l'usage et la temporalité. L'interrelation design-projet est pensée généralement en tant qu'ordonnancement dans le temps. Dès lors, nous nous proposons de penser le rapport design-projet autrement, à partir de la pensée foucaldienne, en proposant de le concevoir en tant qu'un dispositif non normatif avec toutes les dynamiques qui peuvent le bouleverser. Nous tenterons par la suite de rendre compte de l'aptitude de ce dispositif à mettre l'usage au centre de sa dynamique pour le penser en tant que conscience du temps - voire une façon d'être dans le temps -, en s'appuyant sur les propos d'Henri Bergson. Autrement dit, nous souhaitons que ce dispositif ne s'inscrive pas dans la voie des " marchands de temps de conscience » (Stiegler, 2003: 228). Pour ce faire, il est appelé à repenser l'usage, lui ôter son caractère d'usure, ressaisir l'individu hypermoderne d'une attitude consumériste afin qu'il s'approprie un moment (celui de l'usage), qu'il en prenne conscience et le partage avec l'autre.

1 Rihab Zaidi est doctorante et assistante contractuelle à l'ESSTED (École Supérieure des Sciences et Technologies du Design), à l'Université de la Manouba de Tunis. Elle termine une thèse intitulée Le design d'objet. Designer et usager: de l'expérience à la prise de conscience sous la direction du professeur Eric Vandecasteele de l'université de Saint-Étienne ; zaidi.rihab85@gmail.com 


\section{Design et projet : du processus au dispositif}

La notion de projet se positionne dans les fondements de la discipline du design depuis que William Morris et le mouvement Arts E Crafts ont considéré le design en tant que projet de changement social pour lutter contre une « disparition de l'instinct de beauté " (Morris, 2011 : 59). Cette utopie vise à libérer l'homme et la société de l'aliénation machinique. Elle témoigne non seulement de l'engagement de la dualité design-projet mais aussi d'une ambition pour mettre en œuvre une nouvelle pensée de l'objet « convoité " et « conquis » (Morris, $2011: 41$ ), afin de rendre notre monde « un peu plus heureux » (Morris, 2011 : 44). Toutefois, ce projet de " réconciliation » et de changement social que le design a toujours porté dans ses entrailles, est en cours de disparition. Le design s'est plié, selon Hal Foster, aux règles de "l'industrie culturelle» (Foster, $2008: 32$ ) et son projet n'est désormais que le miroir d'une fausse réconciliation. En s'appuyant sur les propos de Jean Baudrillard, Hal Foster nous explique que le design a perdu de vue son projet au cours du temps parce qu'il s'est inscrit dans une logique d' "économie politique de signe » (Foster, 2008 : 32). Ce qui revient à dire qu'il ne s'agit plus de concevoir des objets qui intensifient notre rapport au monde mais plutôt des produits-images destinés à manipuler les désirs d'un consommateur. Dès lors, nous proposons de repenser autrement le rapport design/projet pour saisir les raisons qui font perdre au processus design son sens quand il se confronte à un processus de gestion de projet. Nous nous intéressons au rapport design/projet en tant que «travail de connexion pour construire » (Dubuisson-Quellier et Hennion, 1996 : 22), afin d'introduire un nouvel usage et une nouvelle manière d'être, et dans le monde et dans le temps, étant donné que le temps détermine notre rapport à l'objet.

Nous allons supposer que le design, peut-être «l'un des grands malentendus de l'ère post industrielle» (Vandecasteele, 2007:59), est une sorte d'hybridation entre : un dessin, un dessein et un signe. En effet, Vilèm Flusser (2002 : 7) a tenté de définir le design à partir de la langue anglaise. Selon lui, il signifie " projet, plan, dessein, intention, objectif ", mais aussi " mauvaise intention, conspiration " en tant que substantif. En tant que verbe, to design, signifie " manigancer, simuler, ébaucher, esquisser, donner forme ", et " procéder de façon stratégique ». Il avance l'idée d'un acte de design qui vise à « dé-signer » quelque chose, "lui ôter son 'signe'».

Ceci semble s'écarter de la définition étymologique que Stéphane Vial donne du design qui est selon lui la faculté de « former des signes ou signer des formes » (Vial, $2010:$ 18). Le latinisme du terme « design » nous renvoie au terme « seing » voire la famille du latin « signum » qui signifie une " marque distinctive ». Ainsi, to design voire " désigner » est emprunté au terme latin « designare» qui signifie « marquer d'un signe distinctif» (Picoche, 2011). «En ce sens, écrit Vial, faire du design, ce n'est pas seulement marquer quelque chose d'un signe (signifiant), mais aussi forger un "projet" qui s'incarne dans 
ce signe, c'est-à-dire donner un sens (signifié)»(Vial, 2010 : 19). Toutefois, nous avons le plus grand mal à discerner le sens de ce projet car le design contemporain tend de plus en plus à défier le fonctionnel par le pouvoir de l'imaginaire... Paradoxalement, il projette sans cesse des objets impénétrables submergés par des signes dans un monde postmoderne où tout se joint et communique.

En effet, un projet est, selon la norme NF X50-150, " une démarche spécifique qui permet de structurer méthodiquement et progressivement une réalité à venir » (Digueche, 2014). Nous allons considérer l'objet design comme étant cette "réalité à venir " accomplie, vers laquelle tendent les différentes phases classiques de la gestion d'un projet. Celles-ci peuvent être ramenées à deux grandes périodes : une période de conception, une période de réalisation. La période de conception peut se diviser en deux phases : 1 . étude de faisabilité ; 2 . études préliminaires ou définition. La période de réalisation, elle, peut se diviser en trois autres phases : étude détaillée-développement ; réalisation ; évaluation (Quarante, 1994 : 358).

Pour réduire le temps qui sépare la période de conception et celle de la réalisation, chaque phase doit communiquer ses résultats et se terminer par des choix et des décisions pour déclencher la phase qui suit. Dans un premier temps, la problématique se construit dans une phase analytique, à savoir l'étude de faisabilité qui sera clôturée par un cahier des charges conceptuel. La deuxième phase, qui est en général le terrain le plus familier au designer, est réservée à l'élaboration des propositions et des pré-concepts qui vont déboucher sur un cahier des charges fonctionnel. Dés lors, une étude détaillée s'impose pour mettre en place un cahier des charges technique qui nous amène vers la réalisation, voire la concrétisation du projet. Enfin, il ne reste qu'à effectuer les essais, les suivis et les contrôles.

La gestion du projet requiert toujours différentes méthodes pour contrôler son état d'avancement ainsi que les erreurs et les problèmes qui peuvent surgir à tout moment durant les deux phases. Danielle Quarante les a regroupées en quatre familles : il s'agit des méthodes de gestion de projet, des méthodes de créativité, des méthodes de contrôle de déroulement de l'étude dans le temps et des stratégies de management de l'entreprise. À cet effet, nous constatons que les phases de gestion d'un projet s'inscrivent d'une façon plus générale dans un modèle cyclique qui est celui de la méthode PDCA (Plan : définir des objectifs. $\boldsymbol{D}$ o : les réaliser. Check : comparer la réalisation avec les objectifs. Act : engager des actions d'amélioration), (Autissier, Mougin et Ségot, 2010 : 64). Pour remédier à l'aspect cyclique de ce modèle, Danielle Quarante défend l'idée d'un processus design applicable pour chacune des phases du projet que nous avons déjà définies. Elle le définit en tant qu' " un processus de pensée qui se déroule sur quatre phases : 1- Identification du problème. 2- Analyse. 3- Synthèse. 4- Validation (évaluation)» (Quarante, 1994 : 374). Toutefois, le design a toujours le plus grand mal à se définir malgré toutes les 
représentations et les classifications convenues et logiques qui s'offrent à lui dans le cadre du projet.

Pour notre part, il nous semble que le fait de penser l'acte de design en tant que " processus " pose problème car nous le concevons en tant que succession d'opérations qui visent à produire un résultat. Autrement dit, nous le pensons de la même logique séquentielle que le processus de gestion de projet et nous négligeons son aspect dynamique qui peut catalyser le projet. Il serait peut-être opportun de défendre l'idée d'un espace de liberté que peut générer le rapport design/projet avant de dénoncer la manipulation de l'usager ainsi qu'un possible projet de construction du sujet postmoderne. Il suffit d'observer le recours à la pratique du dessin par certains designers pour comprendre le besoin de se libérer de l'aspect séquentiel du processus. Konstantin Gric (Rubini, 2009 : 11) admet qu'il dessine peu au début du projet et que le dessin lui permet de détailler ses idées quand il passe à l'étape de concrétisation du projet. Naoto Fukasawa affirme quant à lui qu'il sait souvent à quoi va ressembler le résultat final de son projet, mais il a besoin du dessin pour vérifier au fur et à mesure la justesse de ce dernier (Rubini, 2009: 22). Benjamin Graindorge (Rubini, 2009 : 110) évoque un autre rapport au temps dans l'interrelation dessin/dessein. Il conçoit le dessin comme un moyen de contemplation voire un trou dans le temps pour libérer l'esprit puis reprendre le projet. Quant à Jasper Morrison, le dessin lui procure un espace de va-et-vient dynamique entre la feuille et la pensée (Rubini, 2009 : 26).

Dès lors, nous proposons de considérer l'interrelation entre le processus design et le processus projet en tant que dispositif. Nous nous référons de la sorte à un terme incontournable de la pensée foucaldienne. Lors d'un entretien en 1977, Michel Foucault a défini le dispositif en tant qu'ensemble comportant des éléments hétérogènes. Le dispositif est le réseau tissé entre l'ensemble de ses éléments (Agamben, 2007 : 9-10). Il est doté d'une « fonction stratégique dominante » et s' « inscrit dans un jeu de pouvoir » qui met en exergue un croisement de relations pensées et non pas arbitraires. Il importe de souligner de nouveau que nous tentons d'observer de plus près la dynamique qui régit le dispositif design-projet pour le libérer de l'opérationnalité que lui impose le « processus ». Ce n'est pas de l'opposition entre design et projet que nous traitons, mais, plus précisément, de celui du jeu de ressemblance et de complémentarité. Nous n'hésitons pas à nous référer encore une fois à la pensée foucaldienne pour décrypter l'intériorité de ce dispositif. Dans son " archéologie du savoir ", Michel Foucault définit une épistémè pour chaque époque. Afin de rendre visible le rapport de ressemblance du dispositif design-projet, nous allons faire appel à la ressemblance. Celle-ci nous renvoie à quatre différentes formes de similitude telles que Foucault les a énoncées pour le XVI e siècle. Cette forme de savoir "rendait les choses visibles » (Foucault, 1966 : 41). Elle permet de décrypter leurs sens à travers un rapport de ressemblance où « chaque chose trouvera sur une plus grande échelle son 
miroir» (Foucault, 1966 : 46). L'homme se situe ainsi au centre de sa pensée voire « à l'intersection du microcosme et du macrocosme » (Sabot, $2006: 37$ ), à l'instar du dispositif design/projet. Nous allons tenter de saisir ce « rendezvous secret» dans le temps dont nous parle Giorgio Agamben à travers un retour à une épistémè du $\mathrm{XVI}$ siècle, car être contemporain signifie aussi « revenir à un présent où nous n'avons jamais été » (Agamben, 2008 : 36) pour le repenser sous forme d'une archéologie.

Dans un premier temps, le dispositif design-projet peut mettre en place deux choses « convenantes " (Foucault, 1966 : 33). Design et projet seront donc pensés dans un dispositif qui présente un rapport de voisinage et c'est le processus de conception design qui va précéder. Il va définir un problème design qui peut déclencher à son tour le projet. Dès lors, le rapport design/ projet va provoquer un enchaînement continuel des objectifs. Les projets menés par Charlotte Perriand reflètent cette dynamique de voisinage.

En effet, son processus design puise ses idées dans le passé, problématise la tradition japonaise puis l'intègre dans une vision moderne du projet ; tel est le cas de la chaise longue Tokyo qui est une réédition de LCA en bambou. Cette chaise nous renvoie à un modernisme qui respecte, à la fois un savoirfaire artisanal japonais et un héritage culturel pesant. Elle est toujours capable d'intégrer notre espace extérieur en toute confiance, pour lui confier la magie d'une ambiance japonaise telle que la créatrice l'a vécue et l'a insufflée à son objet. La vision moderniste de Charlotte Perriand met en avant un rapport de similitude entre l' " esprit traditionnel japonais ${ }^{2}$ » et la modernité qui s'imprègne de sa proximité avec l'objet et le geste du quotidien.

Cette sympathisante du socialisme a su tisser un rapport de similitude singulier entre design et projet, qui a pris la forme d'une philosophie de la vie apte à garder le passé vivant dans le présent. Toutefois, cette chaîne de la pensée militante, axée sur l'homme, semble être brisée par Louis Vuitton qui prétend détenir un "langage commun ${ }^{3}$ " entre la collection icône du printemps 2014 et les projets de Charlotte Perriand.

Une autre forme de rapport peut notamment régir le dispositif designprojet. Il s'agit du rapport d' " émulation » (Foucault, 1966 : 34). Design et projet sont capables de s'imiter à distance sans voisinage. Plus concrètement, le problème posé à l'origine du projet serait une forme de réduplication du problème design. Le dispositif repose dans ce cas-là sur la réactivité et la compétitivité et non pas l'enchaînement. La collaboration entre l'équipe de designers du Studio Qooq et le métallo Sébastien Bertrand s’inscrit dans cette visée. C'est ce qui fait que « Qooq fait du développement économique par le design chez Art de fer, et inversement Art de fer fait du développement

2 Musée d'art moderne et contemporain Saint-Etienne Métropole. [En ligne]. [Page consultée le 20 avril 2014]. Disponible sur : http://www.mam-st-etienne.fr/data/documents/20130507-EXPO-Perriand-G-Visiteur-web. pdf

3 Youtube. [En ligne], Cassina, 17 Décembre 2013 [Page consultée le 18 avril 2014]. Disponible sur : http:// www.youtube.com/watch?v=eu2ySNSM18k 
économique par l'industrie chez Qooq, l'échange est total » (Fageol et Rivière, 2013 : 85). Ce rapport d' "émulations » instaure une concurrence féconde dans le dispositif design/ projet. Il est apte à perfectionner et équilibrer la surabondance de la production et à favoriser l'échange des compétences et des connaissances.

Une troisième forme de rapport de similitude peut déterminer la dynamique du dispositif, à savoir, l'« analogie» (Foucault, 1966 : 36). Design et projet peuvent déployer des liens de parenté illimités à partir d'un seul point. Ceci est dû au fait que le design est concerné par tout ce qui l'entoure comme l'affirme Jasper Morrison ${ }^{4}$. Ce type de dispositif met en place des ressemblances plus générales à partir des ressemblances partielles. Pour mieux saisir ce rapport, nous nous référons à la collaboration qui a eu lieu entre la chorégraphe Daisy Fel et le Collectif Designer+. Souhaitant aborder la notion d' « intérieur et du rapport des corps avec les objets du quotidien » (Fageol et Rivière, 2013 : 124), la chorégraphe Daisy Fel de la compagnie Litecox, a choisi de rendre hommage à Charlotte Perriand. En essayant d'imaginer le décor de la chorégraphie, elle a exprimé son envie de s' "installer à l'intérieur d'une proposition » (Fageol et Rivière, 2013 : 124). C'est à partir de ce point voire cette ressemblance partielle qui fait appel à la notion d'intérieur, qu'une collaboration entre le Collectif Designer+ et la chorégraphe a été mise en place.

Les deux designers chargées de ce projet, Fabienne Chassier et Lydie Dumassont, n'ont pas hésité à aller à la rencontre des danseurs pour investiguer et s'approprier la scène. Elles sont parties du souci de faire évoluer les corps des danseurs. Le résultat était un cube inspiré de l'univers de Charlotte Perriand, qui se transforme selon l'évolution de l'histoire de la chorégraphie menée par un couple de danseurs. La danseuse Renata en était impressionnée et son témoignage sur cette expérience ne peut que le confirmer : "On était en démocratie, danseurs, chorégraphes, designer... tout le monde s'est exprimé » (Fageol et Rivière, 2013 : 85).

Un jeu de "sympathie-antipathies " (Foucault, $1966: 38-39)$ peut aussi être à l'origine de la dynamique du dispositif design-projet. À ce momentlà, design et projet vont se tenir très proches, mais en même temps distants. $\mathrm{La}$ " sympathie " tend à les fusionner, jusqu'à ce qu'ils soient identiques et perdent leurs singularités. Le dispositif risque de devenir dans ce cas-là une masse homogène dénudée de toute effervescence. C'est l' " antipathie » qui va se charger d'équilibrer ce rapport du moment où elle entretient la différence et préserve la distance. Matali Crasset effleure les limites de ce jeu de «sympathie-antipathie » par la recherche d'une expérience dans notre cadre de vie. Elle part à la quête de l'« objet vivant », voire de l'objet qui peut « donner des potentiels plus que proposer un objet-des possibles, car nous sommes changeants, multiples et évolutifs ». Cette dynamique d'attraction

4 Jasper Morrison [en ligne]. [Page consultée le 30 avril 2014]. Disponible sur : http://www.jaspermorrison. $\mathrm{com} / \mathrm{html} / 03024445 . \mathrm{html}$ 
et de répulsion est pensée par Matali Crasset par le biais des objets qui « se développent dans différents espaces, passent d'une main à l'autre, suivent les différents rythmes de la journée, se transmettent... " (Prodhom, $2002: 6$ ). Quand Jim monte à Paris se veut un concept situé à l'entre-deux du rapport "sympathie-antipathie » où la générosité reflète un rapport au monde qui va au-delà du simple fait d'accueillir quelqu'un chez lui.

Plus concrètement, vers quelle dynamique doit tendre le dispositif designprojet pour ne pas anéantir le processus de gestion du projet par l'urgence de la faisabilité et le processus de conception design par le fardeau de l'actualité ?

\section{l'usage comme une pensée consciente de la temporalité du dispositif Design- Projet}

Dans une interview sur un projet qui consiste à améliorer l'autoroute Saint-Étienne - Givors dans une démarche qui s'appuie sur le design, Olivier Frérot, qui est ingénieur en chef des Ponts-et-chaussées et directeur de l'Agence d'urbanisme de Lyon, déclare qu'il a appris des gens du design de Saint-Étienne que le design est une méthode de conception très profonde, qui a su s'imposer durant ce projet. Il ajoute que «l'ingénieur routier ne met pas les usages au cœur du processus de conception, il met le fonctionnement à travers l'application des normes. Intellectuellement, c'est fondamentalement différent. Cette méthode percute de plein fouet la conception traditionnelle. Quand je parle de conception, cela concerne aussi bien la réparation d'une autoroute ancienne, comme ici, que la conception d'une nouvelle infrastructure » (Frérot et Guilloux, 2006-2007).

Ce témoignage démontre que la période de conception risque souvent d'être dévoyée par l'urgence de la période de réalisation car nous avons tendance à fragmenter et à distinguer les deux périodes, en les pensant en tant que processus alors qu'il faudrait envisager la complexité des deux périodes. Que la temporalité entre en jeu, cela va de soi car design et projet ont déjà en commun le fait qu'ils sont généralement perçus comme des processus de résolution de problèmes, qui se passent dans le temps selon un découpage chronologique.

Cependant, le dispositif design-projet articule deux temporalités différentes, à savoir celles du « dessein » et du « dessin » durant lesquelles l'objet se pense, apparaît, se dessine et se construit. L'objet design n'est pas seulement un simple médium entre designer et usager, qui porte les traces de la phase réalisation d'un projet. Il est plutôt un moyen d'ouverture et sur le monde et sur l'autre. Ceci nous amène à défendre l'idée d'un dispositif centré sur un usage, qui va au-delà de l'objet de consommation, voire de l'objet-instrument (Dagognet, 1989 : 13) que produit le processus de gestion de projet faisant abstraction du processus design. Nous faisons nôtre l'appel de Maurice Merleau-Ponty : "Nous pouvons et nous devons analyser les ambiguïtés de notre temps et 
tâcher, à travers elles, de tracer un chemin qui puisse être tenu en conscience et en vérité » (Ménasé, $2002: 67$ ).

En effet, nous assistons au passage "d'un monde de valeurs stables, à un monde de valeurs variables " (Goux, 2000) dans lequel l'usage risque de devenir un processus d'usure (Arendt, 1993 : 189) et le processus design risque de devenir de plus en plus proche, dans les entreprises, du marketing que de la R\&D (Minvielle, 2011 : 11). D'où, la stratégie de capture de l'usager pour instrumentaliser ses besoins et ses désirs. Ceci écarte toute possibilité d'intégration de l'usage dans le processus de conception comme en témoigne Olivier Frérot. Néanmoins, le dispositif design-projet tel que nous l'avons déjà défini préserve une dynamique. Contrairement au " processus " qui est contrôlé par un temps chronologique axé sur l'actualité et la vitesse, le dispositif design-projet assure une réversibilité du temps. Il permet de retravailler le temps chronologique du processus de l'intérieur pour actualiser par conséquent notre perception de la temporalité de l'usage. De ce fait, l'usage va se libérer d'une vision qui l'associe à une succession de tâches fragmentaires tributaire de la séquentialité d'un temps chronologique. Ce qui revient à dire qu'il importe de réinterroger la temporalité de l'usage.

Sur le fond, il nous semble opportun de signaler que le dispositif mettant en tension le processus design et le processus de gestion de projet pour libérer l'usage et par conséquent l'usager, assure la construction de l'individu. Il met au centre de sa dynamique le sujet ainsi que sa relation au monde qui le construit. C'est pourquoi nous proposons de réfléchir sur une conscience du temps par l'usage, qui permet non seulement d'offrir au dispositif plus de flexibilité, voire même une élasticité du temps mais aussi d'articuler la période de la conception et la période de réalisation par le biais d'une nouvelle temporalité. Pour ce faire, nous sommes appelés à partir des simples " faits » tels qu'ils sont en train de se produire à l'extérieur du dispositif pour comprendre leur «mode de formation » (Bergson, 1967 : 103-104) au sein de ce dernier. Une fois présents dans le réseau d'éléments qui constituent le dispositif, les « faits » seront confrontés à son jeu de pouvoir mais ils vont interagir à des degrés divers avec l'ensemble des éléments. Par ailleurs, l'usage sera synonyme d'usure si nous nous contentons de le penser à partir de l'« extériorité des choses ». Mais à partir du moment où nous parvenons à glisser ces " faits » à l'« intérieur » du dispositif, nous serons aptes à accéder dans un premier temps à " ce qui nous tient intimement, de l'intérieur " (Certeau, $1994: 11$ ) et dans un second temps à « une vie intérieure aux moments bien distincts » (Bergson, 1967 : 103-104). Dès lors, une conscience du temps de l'usage sera possible. Le dispositif centré sur l'usage va emporter «nos états de conscience du dedans au dehors : petit à petit ces états se transforment en objets ou en choses; ils ne se détachent pas seulement les uns des autres, mais encore de nous » (Bergson, 1967 : 103-104). 
Le glissement des « faits » et des «états de consciences » permet de façonner un usage axé sur une prise de conscience, d'une part de la « succession des états " par lesquels passe le dispositif et d'une autre part de ceux qui vont affecter l'acte de l'usage. C'est ainsi que nous pouvons admettre qu'un changement a et aura lieu. Nous nous détachons par conséquent petit à petit de la pensée fragmentaire du temps qui domine l'interrelation design-projet pour accéder à une prise de conscience de la pure durée de l'acte de l'usage. Henri Bergson nous explique que « chacun des états dits successifs du monde extérieur existe seul, et leur multiplicité n'a de réalité que pour une conscience capable de les conserver d'abord, de les juxtaposer ensuite en les extériorisant les uns par rapport aux autres " (Bergson, $1967: 89-90$ ).

Le dispositif design-projet va partir de l'hétérogénéité des "faits » qui forment une "succession des états " pour mettre en place une " homogénéité " de l'espace design-projet. Cette homogénéité favorise la mise en place d'un usage qui dépasse le cadre d'une pensée centrée sur l'« état interne » de la vie individuelle et tend vers un état de conscience qui prend tout son sens dans l'espace social. De même, il est nécessaire de signaler que ce dispositif est appelé à respecter la singularité et la liberté de l'individu car « le fait de donner forme à un projet implique une responsabilité (et donc la liberté) » (Flusser, $2002: 35$ ).

Repenser la temporalité de l'usage de l'objet design revient à repenser aussi «le temps d'apparition des objets » et « le temps de constitution des structures sociales » (Stiegler, 2003 : 221-222) qui leur sont appropriées. Nous sommes loin de sombrer dans une situation de précarité. Toutefois, nous jugeons nécessaire l'engagement du dispositif design-projet dans un processus de libération d'un usager qui a du mal à se définir en tant que " possesseur, ou plutôt possédé» (Dagognet, 1989 : 38). Par-delà leurs différences réelles, design et projet ont au fond un même problème. Tous les deux ont besoin de synchroniser le « je » et le «nous » et de construire puis échanger des usages allant de l'espace intime à l'espace social. De ce point de vue, nous allons supposer que le dispositif design-projet est un "processus d'individuation » dans le temps sachant que le temps n'est que le temps de conscience du "nous » tel que nous l'apprend Henri Bergson.

C'est le temps d'un « qui » et non pas de « quoi » qui génère selon Bernard Stiegler une tension entre le « je » et le «nous». Le « je » est un «potentiel commun » mais il ne faut pas le confondre avec le «nous ». D'où notre volonté de mettre en exergue la prise de conscience de la pure durée de l'usage afin de démontrer l'aptitude du dispositif à conserver non seulement la singularité $\mathrm{du}$ « je » mais aussi celle du " nous ». Cet état de conscience, incarné dans le dispositif design-projet à partir de l'usage, va instaurer une dynamique et un dialogue depuis la conception jusqu'à la réalisation de l'objet design. Désormais, nous pourrons espérer une possible lutte contre l'obsolescence programmée des « objets-fantômes » qui se dissolvent et disparaissent dans 
le brouillard du marché et des exigences démesurées de la société d'hypersollicitation. Cette pensée continuelle de la pure durée de l'usage qui se tisse tout au long du dispositif insuffle un état de conscience à l'usager. Le temps d'un « qui » aura de la sorte un impact direct sur le temps de « quoi ».

Jasper Morrison n'a pas hésitéà expérimenter la temporalité de ses objets pour questionner la façon dont les usages peuvent se réinventer au cours du temps. Il a abordé cette question par le biais du concept de la « supernormalité » qui redonne le pouvoir à la continuité et non pas à l'anticipation et à l'innovation (Jasper Morrison, 2014). Pour avancer une telle idée, Jasper Morrison s'est lancé le défi de faire dialoguer des objets à trois siècles de distance. Il a réussi à intégrer ses objets design dans un même espace avec des objets qui datent du XVIII siècle.

L'exposition Super normal au Musée des arts décoratifs de Bordeaux a dévoilé une vision intemporelle de la notion d'" atmosphère " que nous pouvons aussitôt décrypter dans le récit du designer quand il évoque passionnément ces mises en scènes : "Posée sur un sol en dalles de pierre au milieu de la magnifique cage de l'escalier secondaire, au-dessous de la lampe Glo-Ball d'un diamètre de $45 \mathrm{~cm}$, se trouve une nymphe aux seins nus d'une hauteur d'environ $1,90 \mathrm{~m}$. Sa tête est inclinée et couronnée de fleurs. Ainsi donne-t-elle l'impression d'être tout juste sortie du papier peint posé derrière elle décoré de nymphes - plus petites - en train de distraire Télémaque dans sa quête de l'Odyssée sur l'île d'Ogygie »(Morrison, 2012 : 4). La lampe Glo-Ball a carrément disparu dans le parcours pour ceux qui ne la connaissent pas. Mais ceci ne met pas en doute son « effet de présence » (Beyaert-Geslin, $2012: 50)$.

Pour ceux qui les connaissent, les objets de Morrison ne sont pas passifs. Ils sont aptes à baigner tranquillement dans l'« atmosphère » tout en résistant à une possible dilution dans son homogénéité. La mise en scène ingénieuse de cette exposition est une occasion propice pour expérimenter un effet de résistance par l'usage en tant que conscience du temps. Ces objets qui semblent à première vue projetés dans un ailleurs pour appartenir à un autre temps sont résistants. Ils font croire, dans un premier temps, à ceux qui ne les connaissent pas qu'ils sont normaux et normés voire même absorbés par une collision entre présent et passé. Néanmoins, ils finissent par se démarquer dès que nous acceptons de nous accorder le temps pour nous approcher d'eux, les percevoir, les caresser, les manipuler, etc. L'usage est cet acte qui nous saisit d'une confusion pour vivre une réalité dans l'ici et maintenant. Il nous permet de prendre conscience d'une « succession des états » qui ont été déjà construits dans le dispositif design-projet.

\section{Conclusion}

Rendre conscient est par conséquent une intention et une pensée qui se construisent au cours du dispositif design-projet pour repenser la question de 
la temporalité de notre quotidien matériel. Cette pensée va prendre la forme d'un objet design et non pas la forme d'un « objet-instrument ». Elle repose sur une vision centrale de l' "atmosphère » en tant qu'espace qui peut établir une connexion entre des objets et des individus à partir de leurs différences, et non pas de leur « nature commune » (Arendt, 1993 : 98).

Par ailleurs, nous réinterrogeons la pensée fragmentaire de l'usage en réinterrogeant l'idée du " processus » basée sur la séquentialité et le découpage chronologique. Nous défendons l'idée d'un dispositif Design-Projet centré sur un usage qui se veut une pensée consciente de la temporalité de ce dispositif. Dès lors, nous pouvons souhaiter un possible passage du temps chronologique axé sur l'actualité et la vitesse à une prise de conscience de la pure durée de l'acte de l'usage.

À partir de là, le dispositif design-projet est appelé à saisir non seulement l'ensemble des «faits » et des « états de consciences » qui concerne l'acte de l'usage, mais encore de rendre la conscience de la " succession des états » du dispositif lui-même et de celle de l'acte de l'usage. D'où la possibilité de trouver le bon tempo entre les différents acteurs, méthodes et moyens pour nous livrer une nouvelle temporalité de nos pratiques quotidiennes où la vitesse et l'urgence posent des questions alarmantes. Ceci vise à introduire une réversibilité du temps au rapport design/projet et par conséquent une nouvelle réflexion, et de l'usage et de l'objet design qui va finir par être une partie intégrante de l' « existence consciente » (Bergson, $1967: 97$ ) de l'usager et d'un devenir commun. Penser le projet en tant qu'une construction et non pas une opportunité à saisir, lutter contre la " culture de l'immédiat », telle doit peut être la visée du design contemporain.

\section{BIBLIOGRAPHIE}

AGAMBEN Giorgio (2007), Qu'est-ce qu'un dispositif? Trad. par M. Rueff, Paris, Rivages poche.

AGAMBEN Giorgio (2008), Qu'est-ce que le contemporain? Trad. par M. Rovere, Paris, Rivages poche.

ARENDT Hannah (1993), Condition de l'homme moderne, Paris, Pocket.

AUTISSIER David, MOUGIN Yvon, SEGOT Jacques (2010), Mesurer la performance de la fonction qualité, Paris, Eyrolles.

BARRAL Jacqueline, dir. (2007), Dessein, dessin, design, Saint-Étienne, PU Saint-Étienne.

BERGSON Henri (1967), Essai sur les données immédiates de la conscience, Paris, PUF.

BEYEART-GESLIN Anne (2012), Sémiotique du design, Paris, PUF.

CERTEAU Michel de (1994), L'invention du quotidien 2. habiter, Cuisiner, Paris, Gallimard.

DAGOGNET François (1989), Éloge de l'objet. Pour une philosophie de la marchandise, Paris, Vrin.

DANCER Martine, PERRIAND Pernette et BARSAC Jacques, Musée d'art moderne 
et contemporain Saint-Étienne Métropole. [En ligne]. [Page consultée le 20 avril 2014]. Disponible sur : http://www.mam-st-etienne.fr/data/documents/20130507-EXPOPerriand-G-Visiteur-web.pdf

DIGUECHE Redouane (2014), SCRIBD. [En ligne]. Innovaxion, [Page consultée le 29 mars 2014]. Disponible sur : http://fr.scribd.com/doc/95049477/Qu-est-ce-qu-unprojet

DUBUISSON-QUELLIER Sophie et HENNION Antoine (1996), Le design : l'objet dans l'usage. Paris, Presse des Mines.

FAGEOL Nadine, RIVÈRE Max (2013), Quand le design... devient collectif: 12 projets collaboratifs de designers, Saint-Étienne, Cité du design.

FLUSSER Vilèm (2002), Petite philosophie du design, trad. par C. Maillard, Belfort, Circé.

FOSTER Hal (2008), Design E̋ Crime, trad. par Ch. Jacquet, Paris, Les prairies ordinaires.

FOUCAULT Michel (1966), Les mots et les choses, Paris, Gallimard.

FRÉROT Olivier et GUILLOUX Tristan (2006-2007), «Autoroute design, le design comme méthode de requalification d'une Infrastructure », Flux, 2006/4-2007/1, n 6667 , p. $140-148$.

GOUX Jean Joseph (2000), Frivolité de la valeur, essai sur l'imaginaire du capitalisme, Paris, Blusson.

MÉNASE Stéphanie, (2002) Causerie, Paris, Seuil.

MINVIELLE Nicolas (2011), Design en entreprises. Les pratique de gestion du design, Bruxelles, De Boeck.

MORRIS William (2011), L'art et l'artisanat, trad. par T. Gillybœuf, Paris, Payot et Rivages.

MORRISON Jasper (2012), Jasper Morrison au musée, Paris, Bernard Chauveau.

MORRISON Jasper (2014) [En ligne]. Jasper Morrison LTD. [Page consultée le 30 avril 2014]. Disponible sur : http://www.jaspermorrison.com/html/03024445.html

PICOCHE Jacqueline (2011), Le Robert. Dictionnaire étymologique du français, Paris. (les usuels)

PROD'HOM Chantal (dir.), (2002), Matali Crasset un pas de côté 91/02, trad. par B. Hochsteldt, Paris, Somogy.

QUARANTE Danielle (1994), Eléments de design industriel, Paris, Polythecnica.

RUBINI Constance (2009), Dessiner le design, Paris, Les Arts Décoratifs.

SABOT Philipe (2006), Lire "Les mots et les choses " de Michel Foucault, Paris, PUF.

STIEGLER Bernard (2003), «La désindividuation ». In François ASSHER, Francis GODARD (dir.), Modernité: La nouvelle carte du temps, La Tour-d'Aigues, éd. de L'Aube, p. 221-235.

STIEGLER Bernard (2006), « Du design comme sculpture sociale. Nouvelle association dans les desseins du design ». In Brigitte FLAMAND (dir.), Le design Essais sur des théories et des pratiques, Paris, Éd. du Regard, p. 243-257. 
VANDECASTEELE Eric (2007), «Les fictions du design (nains de jardin et pensée topologique)». In BARRAL Jacqueline (dir.), Dessein, dessin, design, Saint-Étienne, PU Saint-Etienne, p. 59-79.

VIAL Stéphane (2010), Court traité du design, Paris, PUF.

YOUTUBE (2013). [En ligne]. Cassina shares its passion with Louis Vuitton for Charlotte Perriand 17 Décembre 2013. [Page consultée le 18 avril 2014]. Disponible sur : http://www.youtube.com/watch?v=eu2ySNSM18k

Résumé : La présente réflexion n'a pas pour objectif de démontrer ou de remettre en cause l'ordre chronologique «Dessein, dessin, design » (Barral, 2007) où dessein et dessin ont précédé le design. Désirant nous affranchir de l'urgence de la représentation et de la concrétisation qui percute l'interrelation design et projet, nous souhaitons démontrer l'aptitude du processus design à déclencher un processus d' « individuation » dans l'espace du projet afin de mettre en place une nouvelle conception du temps de l'usage d'un objet. Certes l'objet design n'existe que par la pratique du projet mais ce dernier ne doit pas l'emprisonner. Notre objectif vise selon cette perspective à aller au-delà de la matérialité de l'objet en tant que réalisation du projet. Nous proposons de structurer un dispositif design-projet sur une conscience du temps de l'usage. Il s'agit d'ajuster délicatement le faire et la pensée pour atténuer une « oppression du présent »(Certeau, 1994 : 11).

Mots-clés : design, projet, usage, dispositif, temporalité.

\begin{abstract}
The purpose of the present work is neither to prove nor to question the chronological order "Project, drawing, design", where project and drawing have preceded design. In order to avoid the impact that the representation and concretization have on the relation between design and project, we would like to demonstrate that the design process is capable of initializing a process of "individuation" in the project space in order to establish a new way of looking at the use-time of an object. Certainly, the design object exists only through the implementation of the project, but the latter must not restrict the first. Our purpose is, from this perspective, to go beyond the materiality of the object viewed as a concretisation of the project. We propose a model for the design project based on an awareness of the use time of an object. The key is to carefully adjust the doing and the thinking in order to minimize "the oppression of the present».
\end{abstract}

Keywords: design, project, use, device, temporality. 
